\title{
Glucosinolates and isothiocyanates: cancer preventive effects
}

\begin{abstract}
The anticarcinogenic bioactivity of various isothiocyanates (ITCs) has been demonstrated in various animal models such as rats, mice, and hamsters, and in different target tissues such as lung, esophagus, and forestomach. A major chemopreventive mechanism of ITCs involves suppression of reactive metabolite generation and, therefore, a decrease in DNA adducts formation. Many in vitro studies have reported the effects of ITCs on cell cycle modulation and proapoptotic effects through a number of targets, at different points of the pathways. A comparative study in human subjects showed that the hydrolysis of glucosinolates and the absorption of ITCs are greater following ingestion of raw brassica with active plant myrosinase than after consumption of the cooked plant with denatured myrosinase. Apoptosis is considered a vital component of the cellular mechanisms responsible for the maintenance of homeostasis in multicellular organisms. Understanding the pathways of associated proteins provides an insight into the induction of apoptosis by anticancer agents.
\end{abstract}

Keyword: Anticarcinogenic bioactivity; Apoptosis; Cancer preventive effects; Cell cycle modulation; Glucosinolates; Human dietary exposure human; Health isothiocyanates 